\title{
Noise induced hearing loss and other hearing complaints among musicians of symphony orchestras
}

\author{
E. J. M. Jansen • H. W. Helleman • \\ W. A. Dreschler · J. A. P. M. de Laat
}

Received: 14 September 2007 / Accepted: 12 March 2008 / Published online: 11 April 2008

(C) The Author(s) 2008

\begin{abstract}
Objectives An investigation of the hearing status of musicians of professional symphony orchestras. Main questions are: (1) Should musicians be treated as a special group with regard to hearing, noise, and noise related hearing problems (2) Do patterns of hearing damage differ for different instrument types (3) Do OAE have an added value in the diagnosis of noise induced hearing loss (NIHL) in musicians.

Methods 241 professional musicians, aged between 23-64 participated. A brief medical history and the subjective judgment of their hearing and hearing problems were assessed. Musicians were subjected to an extensive audiological test battery, which contained testing of audiometric thresholds, loudness perception, diplacusis, tinnitus, speech perception in noise, and otoacoustic emissions.

Results Most musicians could be categorized as normal hearing, but their audiograms show notches at $6 \mathrm{kHz}$, a frequency that is associated with NIHL. Musicians often complained about tinnitus and hyperacusis, while diplacusis was generally not reported as a problem. Tinnitus was most often localized utmost left and this could not be related to the instrument. It was usually perceived in high frequency areas, associated with NIHL. In general, musicians scored very well on the speech-in-noise test. The results of the loudness perception test were within normal limits. Otoacoustic emissions were more intense with better pure-tone thresholds, but due to large individual differences it can still not be used as an objective test for early detection of NIHL.
\end{abstract}

E. J. M. Jansen $(\varangle) \cdot$ H. W. Helleman · W. A. Dreschler •

J. A. P. M. de Laat

ENT-Audiology, Academic Medical Center Amsterdam,

Meibergdreef 9, Amsterdam 1105 AZ, Netherlands

e-mail: noor.jansen@amc.nl
Conclusions Musicians show more noise induced hearing loss than could be expected on the basis of age and gender. Other indicators, such as complaints and prevalence of tinnitus, complaints about hyperacusis and prevalence of diplacusis suggest that musicians' ears are at risk. Continuing education about the risks of intensive sound exposure to musicians, with the emphasis on the possible development of tinnitus and hyperacusis and the need for good hearing protection is warranted.

Keywords Noise induced hearing loss (NIHL) . Musicians · Tinnitus · Diplacusis · Hyperacusis · Otoacoustic emissions

\section{Introduction}

Noise induced hearing loss (NIHL) is caused by repeated exposure to loud sounds over an extended period of time, exposure to very loud impulse sound(s), or a combination of both. Individuals of all ages, including children, adolescents, young adults, and older people, can develop NIHL, while exposed to intense sounds in the workplace, in recreational settings, or at home. Among the working population who could be affected by NIHL, members of professional symphony orchestras are a specific group for two reasons: they are fully dependent on their hearing for their profession, and they are frequently exposed to loud music. Besides, they have a complicated relation to preventive measures, such as wearing ear muffs or using protective screens, as they may be accompanied by the loss of subtle effects that are necessary to play music and interact with fellow musicians.

In a 1-year noise survey during rehearsals and performances of the Dutch Ballet Orchestra, Boasson (2002) 
found integrated average sound pressure levels that exceed the European guidelines for exposure to sound in a professional environment (a maximum exposure of $80 \mathrm{~dB}$ (A) for $8 \mathrm{~h}$ per day). Boasson also identified four factors that play an important role in the sound pressure levels in orchestra pits: the physical conditions of the orchestra pit, the orchestra arrangement, the repertoire, and the playing time. For many professional orchestras, the acoustical circumstances during practicing or performing are far from optimal with respect to avoiding, or at least minimizing the risks for hearing damage.

NIHL is usually diagnosed by means of the pure-tone audiogram (PTA), the gold standard for identifying hearing threshold levels of individuals, enabling determination of the degree, type, and configuration of a hearing loss. Typical patterns in the hearing thresholds (i.e. a noise notch at 3 , 4 , and/or $6 \mathrm{kHz}$ combined with relatively normal thresholds at $8 \mathrm{kHz}$ ) provide a strong indication for NIHL. Kähäri et al. (2001a, b) showed that the degree of hearing impairment as expressed in the PTA in musicians is smaller than could be expected on the basis of their daily exposure. An extensive review of literature and data of the Vancouver Symphony orchestra concluded that at least some noiseinduced hearing impairment among musicians can be shown from the PTA (Eaton and Gillis 2002). Yet other studies report musicians' hearing threshold levels that do not significantly differ from those of non-exposed populations (e.g. Obeling and Poulsen 1999; Johnson et al. 1985).

The discrepancy between the high number of musicians that report problems with their hearing and their relatively good pure-tone thresholds could partly be explained by selection bias by withdrawal: musicians with hearing problems could have some reservation to participate in such studies. On the other hand, the assessment of musicians' hearing by means of the PTA could lead to very different results than that of, for instance, workers in the building industry. With their well-trained ears and developed sensitivity to sound and music in general (Seither-Preisler et al. 2007), musicians could simply be better in detecting pure tones than other populations.

The measurement of otoacoustic emissions (OAEs) has been proposed to be a more objective and more sensitive test for assessing the effects of noise exposure than the PTA. OAEs are sounds produced by the healthy ear, by the outer hair cells (OHCs) in the cochlea. The absence of OAEs is associated with poorly functioning outer hair cells resulting in reduced selectivity and a decreased sensitivity (e.g. Avan and Bonfils 1993; Gorga et al. 2005; Martin et al. 1990). Lapsley-Miller et al. (2004) found decreased average OAE amplitudes after 6 months of noise exposure, while the average audiometric thresholds did not (yet) change. She found no significant correlations between changes in audiometric thresholds and changes in OAEs, which is suggestive for the hypothesis that OAEs indicate noise-induced changes in the inner ear, still undetected by pure-tone audiometry. When confirmed by further experimental evidence, the measurement of OAEs could be an attractive method to assess NIHL in musicians in an early stage.

Diagnosis of NIHL has often been limited to the measurement of hearing thresholds, while musicians specifically report other sound related hearing problems. Tinnitus (i.e. the perception of noise in the ears or head when no external sound is present), hyperacusis (i.e. an increased sensitivity to loud sounds), distortion (i.e. pure tones are not perceived as pure), and binaural diplacusis (i.e. the pitch of a single tone is perceived differently by the two ears) are among the most often mentioned complaints. Kähäri et al. (2001a, b) already suggested that the way these hearing disorders affect musicians should be investigated further. As these complaints influence a musician's ability to work to full capacity, they should be acknowledged as an important part of a musicians' audiological status and prevention program.

\section{Research questions}

The first question is whether musicians of symphony orchestras should be treated as a special group with regard to hearing, noise, and noise related hearing problems, and whether the instrument type is responsible for different patterns of hearing damage.

Second, the pure-tone audiogram reflects only one aspect of the hearing status of this particular group. The current study aims to obtain reliable, objective data on other expressions of noise related hearing problems: hyperacusis, diplacusis, tinnitus, and decreased performance on speech-in-noise tasks.

The third important issue is the added value of OAE measurements, which are suggested to be more sensitive, more specific, and even more predictive in measuring NIHL. Therefore, we like to assess the relations between measurements of hearing acuity (i.e. PTA, OAE) and selfreports on noise-induced hearing problems.

\section{Methods}

\section{Participants}

A total number of 245 musicians (490 ears) of five symphony orchestras participated in this study on a voluntary basis. Four of them were excluded from the analysis because the severe hearing losses reported in these ears could be attributed to aetiologies other than NIHL. One was removed because of retrocochlear pathology, one due to Menière's disease and two because of asymmetry, not related to noise exposure. 
In total 241 musicians (482 ears) were included in the analyses, 113 females and 128 males between 23 and 64 years of age. In 12 participants not all the tests were performed due to lack of time or because of technical problems in the equipment. The instruments played by the musicians were classified into six groups: high strings (HS): violin and viola; low strings (LS): cello and double bass; wood wind $(W W)$ : oboe, clarinet, bassoon, flute; brass wind $(B W)$ : trumpet, trombone, horn; percussion $(P C)$ and other $(O T)$ : harp, piano, conductor. The distributions of gender, age and instruments are shown in Table 1.

For most participants (i.e. 211, 87\%) it was more than $8 \mathrm{~h}$ ago since they were exposed to music. Some were more recently exposed to noise ( 7 less than $1 \mathrm{~h} ; 8$ between 1 and $2 \mathrm{~h} ; 11$ between 2 and $4 \mathrm{~h} ; 4$ between 4 and $8 \mathrm{~h}$ ). Motorcycle drivers were requested to wear hearing protection during driving and be present at the lab at least half an hour before the tests would start. We decided to include all these participants into the analysis.

\section{Audiological tests}

Participants were subjected to an extended audiological test battery containing tests on audiometric thresholds, loudness perception, diplacusis, tinnitus, speech perception in noise, and otoacoustic emissions. The tests were performed at the ENT-/audiological department of the Academic Medical Centre. Before testing the otoacoustic emissions, the participant had otoscopic inspection in order to check for cerumen. If present, the cerumen was removed by an ENT-doctor.

\section{Audiometric thresholds (PTA)}

Pure-tone air-conduction thresholds at $0.25,0.5,1,2,3,4$, 6 , and $8 \mathrm{kHz}$ were measured using an Interacoustics AC40 audiometer with TDH39 headphones. The audiometer was calibrated according to ISO 389 (1991). Pure-tone measurements were all performed in a sound-isolated booth. Boneconduction thresholds were measured at $0.5,1$ and $2 \mathrm{kHz}$ when air-conduction thresholds exceeded $20 \mathrm{~dB}$. All audio-

Table 1 Distribution of gender and age per instrument category

\begin{tabular}{lllll}
\hline $\begin{array}{l}\text { Instrument } \\
\text { category }\end{array}$ & $\begin{array}{l}\text { Average } \\
\text { age }(\mathrm{SD})\end{array}$ & Gender & \multirow{2}{*}{ Total } \\
\cline { 3 - 4 } & Female & Male & \\
\hline HS & $44(10.6)$ & 64 & 36 & $100(41 \%)$ \\
LS & $48.3(9.4)$ & 16 & 25 & $41(17 \%)$ \\
WW & $42.7(10.6)$ & 25 & 25 & $50(21 \%)$ \\
BW & $43.5(9.9)$ & 6 & 29 & $35(15 \%)$ \\
PC & $43.5(8.9)$ & 0 & 13 & $13(5 \%)$ \\
OT & $41(9.9)$ & 1 & 1 & $2(0.08 \%)$ \\
Total & $44.4(10.2)$ & $112(47 \%)$ & $129(53 \%)$ & 241 \\
\hline
\end{tabular}

metric thresholds were assessed with adequate masking and were expressed in $\mathrm{dB}$ HL, according to standards of diagnostic audiometry.

\section{Loudness perception}

We used an adaptive procedure for categorical loudness scaling ACALOS (Adaptive, Categorical Loudness Scaling) as described by Brand and Hohmann (2002) for three different stimuli: octave-band noises with 0.75 and $3 \mathrm{kHz}$ as the centre frequency, and a wide band noise with a speechshaped spectrum. Each stimulus was presented for $1,000 \mathrm{~ms}$ in a free-field condition. The participant was seated at $1 \mathrm{~m}$ from the speaker producing the noise. For safety purposes, the maximum output was limited to $105 \mathrm{~dB}$ (SPL), according to the JBL control1X specifications. Based on the participant's judgment of the loudness of the test sound for various intensities, an individual loudness curve was fitted. Thus, the dynamic range and the increase of loudness within this dynamic range can be assessed in a single measurement.

\section{Diplacusis}

An adaptive procedure was used to compare the pitch of tonal signals presented alternating to the right and left ear by headphones on three different frequencies: 1,2 and $4 \mathrm{kHz}$. First, participants had to match the loudness of the tone in the left ear to the tone in the right ear, presented at $60 \mathrm{~dB}$ HL. Then, the musician was asked to match the pitch of the tone in the left ear to that of the right ear. Adjustment on the basis of the participants' feedback on both loudness and pitch was done by the test leader, changing the presentation level or the frequency of the tone presented to the left ear in steps of $1 \mathrm{~dB}$ or $1 \mathrm{~Hz}$, respectively.

\section{Tinnitus}

When participants suffered from tinnitus at the time of testing, a tinnitus matching procedure was conducted. First, the tinnitus was localized (i.e. utmost left, left, middle, right or utmost right). In case of clear lateralization, the matching sound was presented to the contralateral ear. When it was localized in the middle, the matching sound was presented to the audiometrically better ear. Then the test leader tried to match the nature of the tinnitus: its character (i.e. pure tone, noise, warble, etc.), pitch, and loudness according to the participant's feedback.

\section{Speech reception in noise (SRT)}

For speech-in-noise testing, we applied a stand-alone version of the telephone test (Smits et al. 2004), installed on a 
laptop computer. The SRT test uses an adaptive procedure, a simple one-up one-down procedure with a step size of $2 \mathrm{~dB}$. Participants responded to each set of three spoken digits (triplets) using the laptop digit-keys. The response was judged to be correct when all three digits were correct. For each SRT measurement a series of 23 triplets is chosen randomly out of 80 triplets: the SRT was then calculated by averaging the signal-to-noise ratios of the last 20 presentation levels (i.e. the last presentation level is based on the last response).

\section{Otoacoustic emissions (OAEs)}

Both transient evoked otoacoustic emissions (TEOAE) and distortion product otoacoustic emissions (DPOAE) were measured on both ears of each musician using Otodynamics ILO 292 equipment. Each test day the probe was calibrated before OAE-measurement.

TEOAE's were evoked using a $80 \mathrm{dBpeSPL}$ click stimulus. They were measured in the non-linear mode and filtered in half-octave frequency bands at 1, 1.5, 2, 3 and $4 \mathrm{kHz}$.

DPOAE were evoked using pairs of tones $f_{1}$ and $f_{2}$ with particular intensity and frequency relations $\left(f_{1}: f_{2}\right.$ ratio). The evoked response from these stimuli occurs at a third frequency, the distortion product frequency $f_{\mathrm{dp}}$, which is calculated as $f_{\mathrm{dp}}=2 \times f_{1}-f_{2}$. The DPOAEs levels of the primary tones, $L_{1}$ and $L_{2}$, were 75 and $70 \mathrm{~dB}$ SPL, respectively. The frequency ratio of $f_{2} / f_{1}$ was 1.22 . DPOAEs were measured at the frequency $2 f_{1}-f_{2}$ for $27 f_{2}$ frequencies ranging from 815 to $8,000 \mathrm{~Hz}$ (i.e. 8 points per octave). The emission level was established on the basis of three presentations. In case of high noise floors, the measurement was repeated manually at particular frequencies, usually below $2 \mathrm{kHz}$.

\section{Questionnaire}

All participants completed a self-report questionnaire that consisted of the relevant questions related to ear and hearing problems in the medical history, questions about behaviour towards loud music and noise, questions about personal hearing complaints, the use of hearing protection, and subjective judgments of own hearing capacity.

\section{Statistical analyses}

All statistical analyses were performed using SPSS 12.01.

Part of the data has been obtained per ear (i.e. pure-tone thresholds, OAE-responses). In that case, some detailed analyses were performed per ear. However, the majority of results were considered per participant. When these data were compared to data obtained per ear, average scores over the ears were used.

Audiogram data usually have a skewed (i.e. positively slanting) distribution as hearing thresholds increase rather than decrease. We assumed that our tested sample was large enough to approach a normal distribution, so we could use parametric tests for the audiometric data (Dawson-Saunders and Trapp 1994).

Data which were obtained per ear (i.e. audiometric-, and OAE-data) on various frequencies were tested using a general linear model (GLM) Repeated measures ANOVA. Differences on separate audiometric frequencies were tested with a MANOVA over ears.

Data that were obtained on individuals (i.e. data on loudness perception, and speech-reception thresholds in noise), or in combination with the audiometric data were analysed using paired sample $t$ tests, and bivariate correlations.

The significance level used for all the tests and the correlations was $p=0.05$ or smaller.

Data on frequencies (e.g. diplacusis, tinnitus, self-report data, etc.) were analysed using non-parametric tests (Kruskall-Wallis, Chi-square) with a similar significance level $(p<0.05)$.

The focus is on the following results:

- The status of the hearing of musicians as compared to a general population.

- The specific subjective complaints of musicians in relation to objectively measurable facts.

- The differences between musicians in the previously defined instrument categories.

Whenever possible, we compared our data to that of known population numbers. In analyses over instrument categories, percussion (PC) and other (OT) were not included as the number of musicians in these categories did not exceed 20. Where relevant, the results of the percussionists will be discussed qualitatively.

\section{Results}

Effects in the pure-tone audiogram

A vast majority of the musicians (92\%) reported healthy ears. Forty-one $(17 \%)$ indicated to have suffered from ear infections in childhood. Sixty-five (27\%) ever visited an ENT-doctor for complaints about their hearing. Eighty-nine $(37 \%)$ indicated hearing problems in the family, mostly related to presbyacusis. No association with ear infections in early childhood and the presence of hearing problems in the family could be found in the data set. 
NIHL is generally associated with a notch-shaped highfrequency sensorineural loss that is worst at $4 \mathrm{kHz}$, but the notch often occurs at 3 or $6 \mathrm{kHz}$ as well (e.g. Coles et al. 2000). There have been several attempts to identify audiometric notches according to objective criteria (Coles et al. 2000; Rabinowitz et al. 2006; Niskar et al. 2001). In these studies, audiograms are usually divided in normal hearing, age related hearing loss, and noise induced hearing loss. Applying these criteria, most of the audiograms of our musicians would be identified as normal hearing, a few as NIHL and some as age related hearing loss. As we would like to get more insight in the development of the musicians' hearing (i.e. the noise notches) over time (in view of a follow-up study) and the possibility to relate the audiograms to otoacoustic emissions, we aimed at a more detailed differentiation in noise notches. Therefore, we used a rather strict criterion for "normal hearing", and more specific criteria for the degree of the noise notch. The following audiogram categorization was applied to the audiometric thresholds per ear:

- Normal hearing $(N)$ : hearing threshold levels better than or equal to $15 \mathrm{~dB} \mathrm{HL}$ at all measured frequencies (i.e. 0.5 , $1,2,3,4,6,8 \mathrm{kHz})$.

- Notch moderate (NM): maximum threshold level of 3, 4, and $6 \mathrm{kHz}$ between 15 and $20 \mathrm{~dB}$ poorer than the puretone average of thresholds at $0.5,1$ and $2 \mathrm{kHz}$ and at least $10 \mathrm{~dB}$ poorer than the threshold level at $8 \mathrm{kHz}$. This is similar to Niskar et al. (2001) criterion of a noise notch in adolescents.

- Notch profound (NP): similar to NM, but maximum threshold level of $3,4,6 \mathrm{kHz}$ at least $25 \mathrm{~dB}$ poorer than the pure-tone average of thresholds at $0.5,1$ and $2 \mathrm{kHz}$.

- Sloping loss (SL): maximum threshold level of 3, 4, $6 \mathrm{kHz}$ at least $5 \mathrm{~dB}$ poorer than the pure-tone average of thresholds at $0.5,1$ and $2 \mathrm{kHz}$ and threshold level at $8 \mathrm{kHz}$ at least $5 \mathrm{~dB}$ poorer than the maximum threshold level at 3, 4, and $6 \mathrm{kHz}$.

- Flat loss (FL): audiograms which do not fall into the above mentioned categories, with no hearing thresholds exceeding $30 \mathrm{~dB}$ at all measured frequencies.

- Rest (R): all audiograms that do not match the characteristics of the above described categories.

The corresponding average audiograms are shown in Fig. 1. The average audiogram in the group "Rest" turned out to have a steeply sloping curve. Most ears fell in the "Normal hearing" category (230 ears, 48\%). The other ears were approximately equally divided over the other categories $(\mathrm{NM}=53$ ears, $11 \%, \mathrm{NP}=41$ ears, $9 \%, \mathrm{SL}=64$ ears, $13 \%$, $\mathrm{FL}=57$ ears, $12 \%, \mathrm{R}=35$ ears, $7 \%$ ). If present, notches were mostly found at $6 \mathrm{kHz}$.

In the "Normal hearing" category the average age of the ears was lowest (39.7 years), while it was highest in the

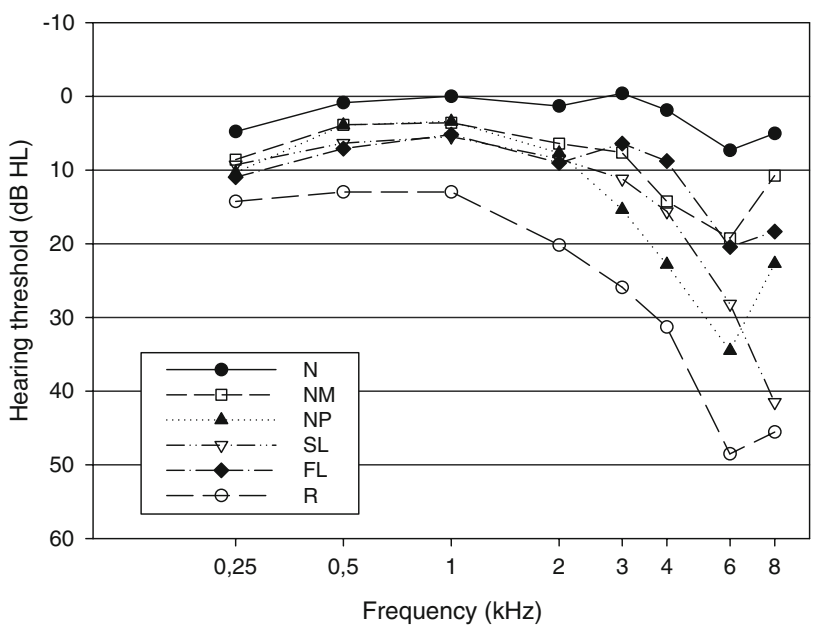

Fig. 1 Musicians average audiograms according to the criteria for normal hearing $(N)$, notch moderate $(N M)$, notch profound $(N P)$, sloping loss $(S L)$, flat loss $(F L)$, and a rest group $(R)$

"Sloping loss" category (52.2 years). For the category "Notch profound" (48.8 years) it was higher than for the category "Notch moderate" (45.1 years). A direct comparison of the distribution of audiometric categories across instruments groups could only be done with some caution, as there were large variations in the number of musicians in the instrument subgroups. However, when considering only the large groups, HS, LS, WW and BW, 40-52\% of each of these groups fell into the audiogram category "Normal Hearing". The percentages did not differ significantly $\left(\chi^{2}(3)=2, p=0.57\right)$. Hearing loss with sloping curves (SL) was found less among the brass wind players (2 ears, $3 \%$ ) than in the other groups (HS $=28$ ears, $14 \%, \mathrm{LS}=16$ ears, $20 \%$, and $\mathrm{WW}=13$ ears, $\left.13 \%, \chi^{2}(3)=11.9, p=0.007\right)$.

In the other audiogram categories (NM, NP, and FL) no significant differences were found across large instrument groups (tested with $\chi^{2}, p>0.05$ )

Absolute threshold levels, as used thus far, facilitate the comparison of the PTA threshold levels with the results of other audiological tests. Absolute pure-tone thresholds are, however, known to be strongly dependent on age and gender. Therefore, we also calculated relative thresholds, corrected for gender and age effects according to ISO 7029 (2000) standards. Relative thresholds were derived by subtracting the population median. Next the percentages of ears that were above the P90, P75, median, P25, and P10 percentile points were generated. The results are presented in Fig. 2.

In Fig. 2, the relative audiometric results of the musicians are presented by dotted lines, in which the symbols refer to the corresponding percentile values. The drawn lines correspond to the ISO-population percentile scores. When the musicians would have had a normal distribution of hearing levels according to age and gender, the dotted 


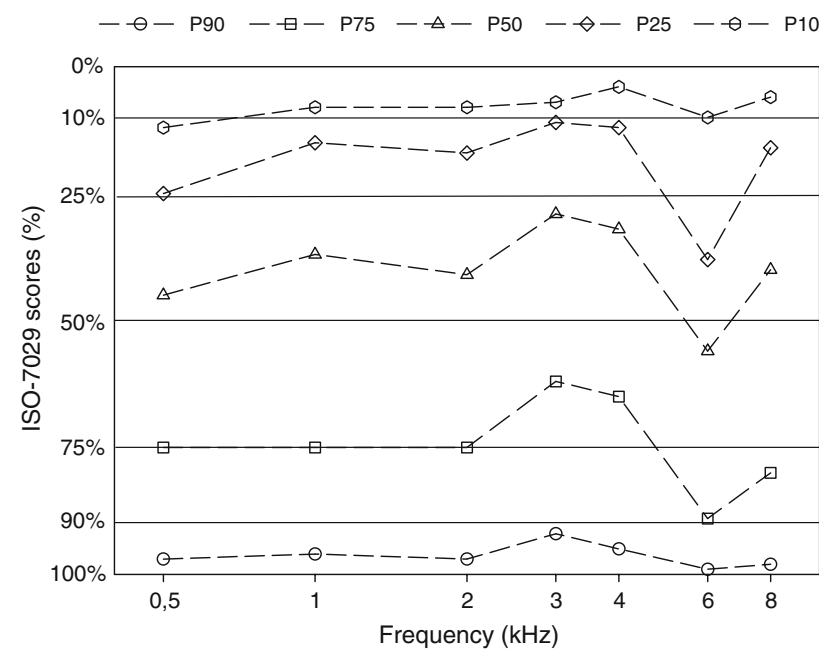

Fig. 2 Relative (i.e. corrected for age and gender) median and percentile scores as opposed to ISO 7029 (2000). Continuous lines represent a population according to ISO 7029 (2000), dotted lines represent the musicians' scores of this study

lines would coincide with the drawn lines as is the case for the 75 th percentile line at $0.5,1$, and $2 \mathrm{kHz}$. At the 10 th percentile, the 25 th, the 50 th, and the 75 th percentile a large number of musicians score equal to or better than the ISOpopulation at all frequencies, except at $6 \mathrm{kHz}$ where the distribution of thresholds is shifted relative to the ISO-population. The 90th percentile of the musicians is placed beneath the 90th percentile of the ISO-population at all frequencies. The figure clarifies that the distribution of hearing thresholds in musicians - after a correction for age and gender is generally more favourable than would be expected on the basis of ISO 7029 (2000), except at $6 \mathrm{kHz}$, at which a higher percentage of the musicians scored below the ISOpercentile scores. These results strongly suggest that NIHL occurs more often in musicians than in the ISO-reference population.

A GLM repeated measures analysis over the relative thresholds per ear at all frequencies, showed that the instrument played by the musicians (analysed for the large subgroups HS, LS, WW, and BW) affected the distribution of relative average thresholds $(F(3,439)=419.8, p=0.04)$. A post-hoc test (LSD) showed that the average relative threshold of low-string players (LS) was significantly better than the average relative threshold of high-string (HS), wood-wind (WW) and brass-wind (BW) players ( $p=0.019, p=0.019, p=0.012$, respectively). In Fig. 3, the relative audiometric thresholds per instrument category are shown.

\section{Other symptoms of NIHL}

In this section, all results have been analysed per participant. For comparison between the results in these tests and

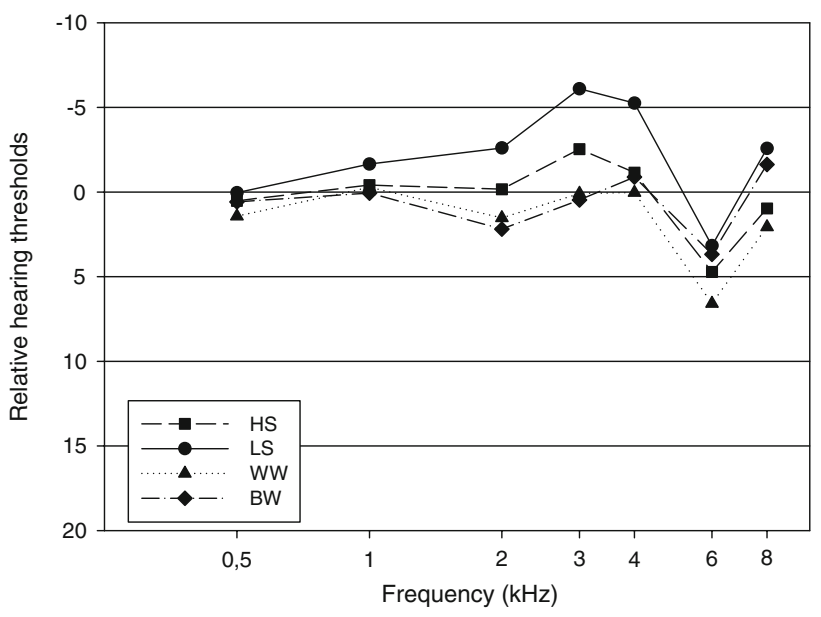

Fig. 3 Average relative (i.e. corrected for age and gender) audiograms for instrument categories

the pure-tone thresholds, we used the average pure-tone thresholds of both ears.

In the analysis of loudness perception, the focus was on the uncomfortable loudness level (UCL) and the dynamic range (DR). The UCL is the level at which a stimulus is perceived as uncomfortably loud. It can provide information about the sensitivity for loud sounds and in that sense it is related to hyperacusis. A sum of 239 musicians participated in the loudness perception test. Their UCLs ranged from 76 to $120 \mathrm{~dB}$ SPL and the average UCL values were slightly lower than could be expected on the basis of the UCLs at pure tones in a general population. The average values were 103,100 , and $105 \mathrm{~dB}$ SPL for $0.75 \mathrm{kHz}$ NBN, $3 \mathrm{kHz}$ NBN, and WBN, respectively. These differences all were significant when analysed by paired $t$ tests. Consequently, the $3 \mathrm{kHz} \mathrm{NBN}$ was perceived as the least comfortable stimulus and the WBN as the most comfortable.

The DR is the range between the just noticeable stimulus intensity (i.e. usually close to the pure-tone threshold, at critical unit 5) and the intensity of the stimulus at the UCL (i.e. critical unit 50). The DR covers 45 critical units and provides information about the range in which a person can hear properly. This is strongly related to the phenomenon of recruitment that usually accompanies hearing loss from a cochlear origin. The DRs ranged from 48 to more than $120 \mathrm{~dB}$ (i.e. the maximum levels allowed) with average values of 82,79 , and $82 \mathrm{~dB}$ for $0.75 \mathrm{kHz} \mathrm{NBN}, 3 \mathrm{kHz} \mathrm{NBN}$, and WBM, respectively. The DRs at $3 \mathrm{kHz}$ NBN differed significantly from the DR at $0.75 \mathrm{kHz} \mathrm{NBN}(p<0.001)$ and WBN $(p<0.01)$. We found no significant difference in the DRs of $0.75 \mathrm{kHz}$ NBN and WBN.

The DRs showed a number of significant correlations with the average absolute pure-tone threshold of both ears at $1,2,3,4,6$, and $8 \mathrm{kHz}$, showing a decreasing DR for increasing pure-tone thresholds, but all correlations were weak (all $r^{2}<0.09$ ). 
In the results of the diplacusis matching the deviation between the ears is expressed as a percentage of the measured frequency (e.g. when the pitch of a $1,000 \mathrm{~Hz}$ tone presented to the right ear is matched to the pitch of a $1,333 \mathrm{~Hz}$ tone presented to the left ear, the outcome measure is $3.3 \%$ ). Table 2 shows the numbers and percentages of musicians with an interaural pitch difference of more than 1,2 , or $3 \%$, respectively, and the numbers and percentages of musicians per instrument category that show diplacusis to such degrees. For a total of 106 musicians (44\%) the interaural pitch difference was more than $1 \%$, for 43 (18\%) it was more than $2 \%$, and for $20(3 \%)$ more than $3 \%$ at one or more of the tested frequencies. Diplacusis more often occurs in the higher frequencies.

Although the prevalence of diplacusis seemed to be higher among WW and BW-players, no significant differences in the degree of diplacusis at 1,2 , and $4 \mathrm{kHz}$ were found between instrument categories ( $\chi^{2}$ test, $\left.p>0.05\right)$. There was no significant age effect.

A small but significant correlation was found between the asymmetry in the pure-tone audiogram and the perceived pitch difference at $4 \mathrm{kHz}(r=0.22, p=0.001)$. The pitch of the $4 \mathrm{kHz}$ tone tended to be perceived lower in the ear with the poorest threshold. Participants with an interaural difference of $1 \%$ or more at 1 and $2 \mathrm{kHz}$ had significantly higher pure-tone thresholds [resp. $F(1,223)=7.6$, $p=0.006, F(1,233)=6.35, p=0.012)]$.

Tinnitus matching could only be performed in case the tinnitus was present at the moment the test was taken. Accordingly, 42 (17\%) musicians participated in this test. The level of the tinnitus was matched and compared with the audiometric threshold levels resulting in a sensation level of the matched tone (dB SL). On average the sensation level of the tinnitus was $4 \mathrm{~dB}$, but it ranged from 0 to $32 \mathrm{~dB}$ SL.

In a number of cases, it was difficult to match the character of the tinnitus with the audiometer sounds. Qualitative descriptions most often showed a high pitched tone-like sound, but numerous variations were mentioned (e.g. warble, hiss, buzz, ring, waterfall, crackle, vague tone, etc.). Pitch was matched with pure tones between 0.125 and $8 \mathrm{kHz}$. Ten participants $(25 \%)$ indicated the pitch of their tinnitus was lower than $4 \mathrm{kHz}$. A sum of 15 participants $(35 \%)$ indicated a pitch between 4 and $8 \mathrm{kHz}$. Unfortu- nately, we could not estimate pitch above $8 \mathrm{kHz}$, as 17 (40\%) musicians indicated a pitch higher than $8 \mathrm{kHz}$.

Tinnitus was more often localized utmost left $(18,43 \%)$ than utmost right $(7,17 \%)$ and middle $\left(13,31 \%, \chi^{2}(4)=\right.$ $38.1087, p<0.001)$. However, no significant difference in localization was found between the instrument categories $(p>0.05)$. There was no significant effect of gender.

Participants with tinnitus at the moment of the test had significantly worse average pure-tone thresholds than the ones without tinnitus at the moment of the test $(F(1$, $231)=18.51, p=0.03)$. This was especially the case for the higher frequencies. Not surprisingly, the average age of the participants with tinnitus at the moment of the test was also higher $($ mean $=43.3$ vs. mean $($ tinnitus $)=50.8, F(1,231)=$ $18.34, p<0.000)$.

A total of 239 musicians participated in the speech-innoise test. The average speech-to-noise ratio (SNR) was -6.7 (SD 1.4), ranging from -9.2 to -1.6 . The majority of participants $(231,96.6 \%)$ scored an average SNR lower or equal to -4.1 , indicating good hearing. 8 (3.3\%) participants scored an SNR between -4.1 and -1.4 (i.e. moderate hearing). No participants scored higher than -1.4 , indicating poor hearing as defined by Smits et al. (2004).

No significant differences were found between the mean SNRs for the factors instrument category, age, or gender.

The correlation between the SNR and the pure-tone thresholds at all measured frequencies was relatively low, but highest and significant at $3 \mathrm{kHz}(r=0.26, p<0.001)$.

The questionnaire

Most often the musicians judged their hearing of 10 years ago as significantly better than 5 years ago, while the latter was rated as significantly better than their hearing now (mean: 8.8 vs. 8.2 vs. 7.6 Wilcoxon signed ranks tests $p<0.01$ ).

When asked to judge the quality of one's own hearing in quiet, in noisy environments and when making music, no significant differences were found in these situations (these ratings were performed on a scale from 1 (very poor) to 5 (very good).

A sum of $46(19 \%)$ of the musicians indicated they would be ashamed of having hearing disorders. When asked to further clarify their answer, $12(5 \%)$ thought they

Table 2 Distribution of diplacusis over frequencies and instrument categories

\begin{tabular}{|c|c|c|c|c|c|c|c|}
\hline \multirow[b]{2}{*}{$\Delta$ freq ears } & \multicolumn{3}{|c|}{ Number and $\%$ on tested frequencies } & \multicolumn{4}{|c|}{ Number and $\%$ musicians per instrument category } \\
\hline & $1 \mathrm{kHz}$ & $2 \mathrm{kHz}$ & $4 \mathrm{kHz}$ & $\mathrm{HS}$ & LS & WW & BW \\
\hline$>1 \%$ & $29(12 \%)$ & $41(17 \%)$ & $72(30 \%)$ & $39(39 \%)$ & $14(33 \%)$ & $30(60 \%)$ & $16(46 \%)$ \\
\hline$>2 \%$ & $7(3 \%)$ & $17(7 \%)$ & $2912 \%$ & $14(14 \%)$ & $6(14 \%)$ & $23(46 \%)$ & $8(23 \%)$ \\
\hline$>3 \%$ & $1(0.4 \%)$ & $5(2 \%)$ & $14(6 \%)$ & $6(6 \%)$ & $3(7 \%)$ & $6(12 \%)$ & $3(9 \%)$ \\
\hline
\end{tabular}

Values are expressed in numbers and percent. 
would not be a good musician in case of hearing problems, $6(2 \%)$ stated that they thought their colleagues would doubt their ability to function as a musician. This made some participants reluctant to talk about it or to take measurements associated with hearing problems (i.e. for some this also included wearing hearing protection). A few (16/ $7 \%$ ) stated they were afraid of losing their job after the orchestra management would be informed about hearing problems. A sum of $6(2 \%)$ thought this question was not applicable to them (i.e. because they did not suffer from hearing complaints), and 20 (8\%) thought hearing problems are part of the life of a musicians and should therefore be discussed in all circumstances.

A large number of musicians indicated to use hearing protection: $152(52 \%)$ during orchestra repetitions, 70 (29\%) during concerts and $87(36 \%)$ during other occasions, such as visits to a discotheque and other leisure activities. Females indicated to wear hearing protection more often than males during repetitions and concerts $\left(\chi^{2}(1)=4.68, p=0.03\right)$. A few musicians only wear hearing protection when strictly necessary and only in one ear (e.g. the ear on the side of percussion or brass winds). Most wearers use disposable hearing protectors (foam or cotton), a few have custom-made hearing protectors.

When asked about other auditory deficits (i.e. hyperacusis, diplacusis, tinnitus, and distortion) 190 (79\%) reported complaints about hyperacusis, 17 (7\%) about diplacusis, $121(51 \%)$ about tinnitus, and $57(24 \%)$ about distortion of tones. The degree of the complaints varied from slight to severe. Figure 4 shows cumulative results on the five-point rating scale. The number of musicians that suffered from hyperacusis, diplacusis, tinnitus, or distortion did not depend on the instrument played by the musician or gender $(p>0.5)$.

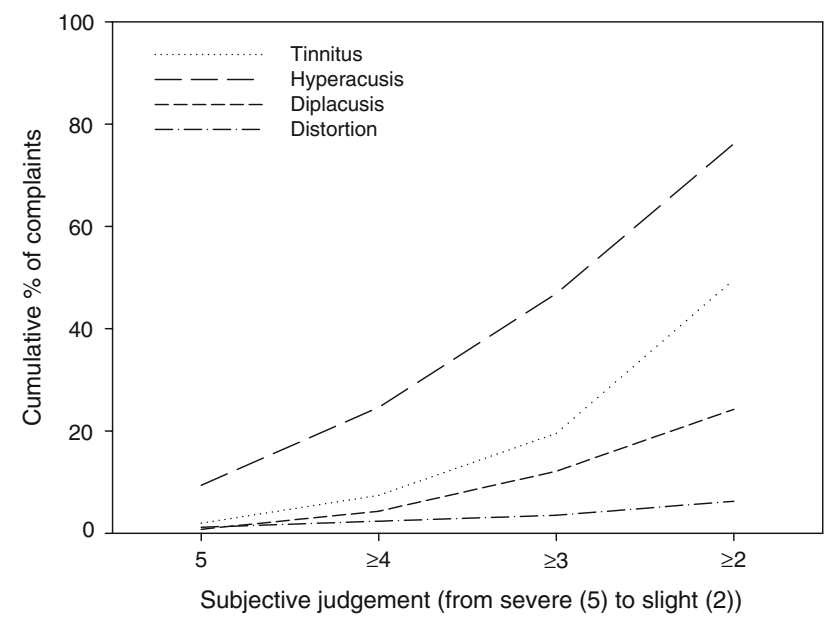

Fig. 4 Cumulative proportion of musicians with complaints (ranging from slight to severe) about tinnitus, hyperacusis, diplacusis and distortion
Hyperacusis was mentioned most frequently as an auditory problem. A total of $79 \%$ indicated to be sensitive to loud sounds varying from slight $(52,22 \%)$ to very severe $(23,10 \%)$.

When comparing the subjective complaints about hyperacusis with the results of the loudness-perception test, a small, but significant correlation was found: musicians who indicated to suffer severely from hyperacusis scored slightly lower UCL's in the loudness perception test than others who indicated no or mild suffering $(r=-0.29$ for $0.75 \mathrm{kHz} ; r=-0.21$ for $3 \mathrm{kHz} ; r=-0.15$ for WBN, $p<0.01)$. No significant differences were found between the large instrument groups. Females, however, indicated to suffer from hyperacusis more severely than males $\left(\chi^{2}(4)=10.3, p=0.04\right)$.

Only $7 \%$ of the musicians indicated to experience an interaural difference in pitch perception in contrast to the results of the diplacusis matching where $18 \%$ showed an interaural pitch difference of more than $2 \%$. When the subjective results on the question of diplacusis were compared to the results of the diplacusis matching, no significant correlation was found for any of the tested frequencies. No significant difference was found between males and females on the subjective rating of diplacusis.

One hundred and thirty two (51\%) musicians indicated to have complaints about tinnitus, varying from slight (42, $32 \%)$ to severe $(3,2 \%)$.

The large instrument groups (i.e. HS, LS, WW, BW) showed only slight differences in the number of participants with tinnitus. Tinnitus occurred the least in low string players, while it occurred more often in brass wind and high string players. No gender difference was found in the subjective rating.

\section{Effects in OAE-responses}

OAE-responses were obtained from 479 ears. Large interindividual differences were found in TEOAE responses of the musicians in all frequency bands $(1,1.5,2,3$, and $4 \mathrm{kHz}$ ) and the median intensity levels of the TEOAE were slightly decreasing with increasing frequency. In a GLM repeated measures analysis with gender as between subjects factor, and frequency band as the repeated measure, females show overall higher TEOAE-responses than males (average response over all frequencies 8.4 vs. 4.6, $F=8.9$, $p<0.001)$. No significant differences were found for TEOAE-responses between the left and right ear $(p>0.05)$.

Taking only the large instrument categories (i.e. HS, LS, WW and $\mathrm{BW}$ ) into account, the instrument significantly affected the overall TEOAE response $(F(4,4)=3, p<0.01)$ : brass wind players showed the lowest responses and high- and low-string players the highest. Responses covariated 
with age $(F=3.5, p<0.01)$ showing decreased responses with increasing age.

DPOAE responses showed the characteristic DPOAE configuration over the 27 tested frequencies (i.e. an increase in DPOAE response in the lower and higher frequencies and a decrease in the middle frequency levels), and a large inter-individual variation, especially beneath the median. Again, females show stronger intensity levels than males, especially in the higher frequency regions (average response over all frequencies 8.0 vs. 5.6, $F=16.5$, $p<0.001)$. When including only the large instrument categories (i.e. HS, LS, WW, BW) into the analysis, we found significant differences in DPOAE responses $(F(3,26)=$ $3.14, p<0.01)$ : High- and low-string players showed overall higher DPOAE responses than wood-wind and brasswind players. No significant interactions were found with gender and instrument category $(F=1.2, p>0.5)$. The DPOAE intensity levels also covariated with age, showing a decrease in intensity with increasing age $(F=4$, $p<0.001$ ).

TEOAE and DPOAE responses significantly correlated at the same frequencies $(1,1.5,2,3$, and $4 \mathrm{kHz}): R^{2}$ ranged from 0.27 to $0.45, p<0.001$.

The individual relation between TEOAE and DPOAE responses and the pure-tone thresholds was weak. Some musicians showed (almost) normal pure-tone thresholds with surprisingly low OAE responses, while others showed poor pure tone thresholds, but relatively high OAE responses. Correlation coefficients between audiometric thresholds and TEOAE intensity levels at the same frequencies were significant, but low: $R^{2}=0.17 / 0.19 / 0.22 / 0.23$, $p<0.05$ ) at $1,2,3$, and $4 \mathrm{kHz}$, respectively. The correlation between the average TEOAE response and the average pure-tone threshold at $1,2,3$, and $4 \mathrm{kHz}$ was 0.29 . Slightly higher correlations were found between the DPOAEresponses and the pure-tone thresholds: $R^{2}=0.13 / 0.21 /$ $0.37 / 0.40$ at $1,2,3$, and $4 \mathrm{kHz}$, respectively and $R^{2}=0.45$ for the average pure-tone threshold and average DPOAE response of 1,2, 3, and $4 \mathrm{kHz}$.
In addition to the individual data, we also investigated the OAE distributions in the audiogram categories defined above. The average TEOAE per audiogram category is shown in Fig. 5a, the average DPOAE in Fig. 5b. The figures illustrate that the musicians in the normal hearing category have the strongest overall TEOAE (mean $=8.04$, $\mathrm{SD}=4.6$ ) and DPOAE (mean $=9.51, \mathrm{SD}=4.6$ ) responses, while musicians in the rest category show the weakest TEOAE $($ mean $=3.32, \mathrm{SD}=5.7)$, and DPOAE (mean = $2.01, \mathrm{SD}=6.6$ ) responses. Significant differences were also found between OAE of the normal hearing category and the other categories (i.e. N vs. NM, NP, SL, and FL, post-hoc Bonferroni, $p<0.05$ )

\section{Discussion}

The first experimental question was whether musicians of symphony orchestras should be treated as a special group with regard to hearing, noise, and noise related hearing problems.

A combination of factors puts the hearing of many professional musicians at risk: they are often subjected to intense sound levels for long periods of time, while studying, rehearsing, and performing music. They often play in acoustically poorly equipped spaces (Boasson 2002) and many feel disabled when wearing hearing protection as it affects the way they play and hinders interaction with colleagues.

Studies on the hearing of musicians in symphony orchestras have indicated that their pure-tone hearing thresholds do not really deviate from that of a nonexposed population (e.g. Kähäri et al. 2001a, b; Eaton and Gillis 2002; Obeling and Poulsen 1999). It has been hypothesized that specific "musician characteristics" are responsible for this result: wanted sounds such as music could be less harmful than unwanted sounds such as industrial noise (Karlsson et al. 1983), or musicians perform relatively good on pure-tone audiometry because of
Fig. 5 a Average TEOAEintensity levels for musicians in each audiogram category b Average DPOAE-intensity levels for musicians in each audiogram category

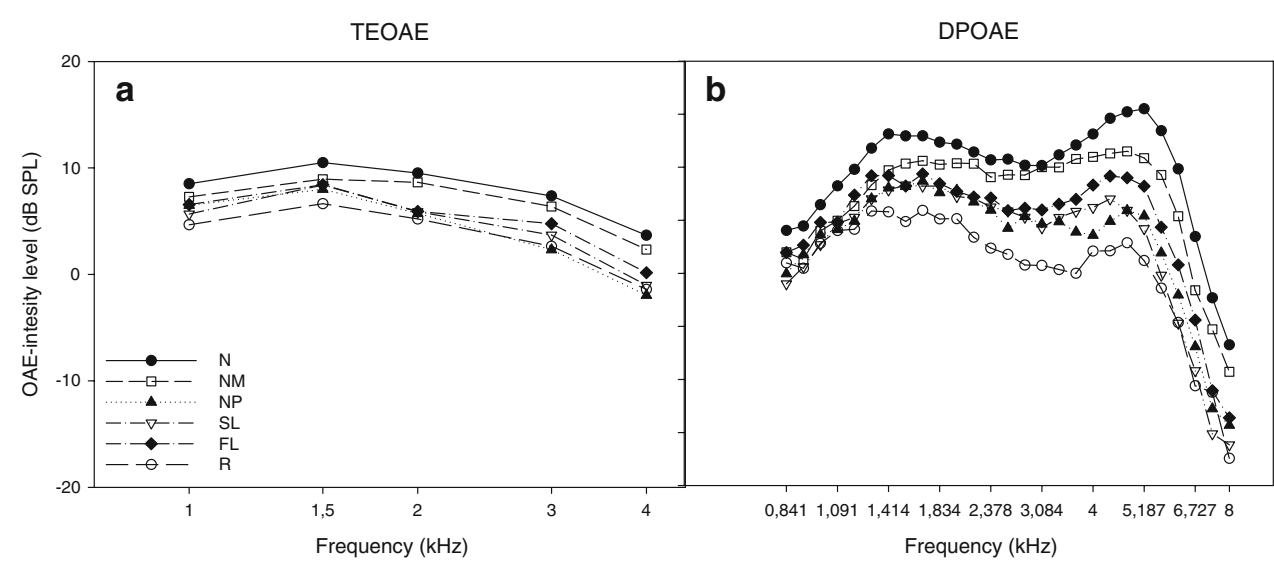


a strong motivation and familiarity with detecting pure tones (Dowling and Harwood 1986).

The musicians participated on a voluntary basis. We are aware that this could have produced a selection bias, probably towards the better hearing musicians, as musicians with hearing complaints may have been reluctant of having their hearing tested. Most musicians judged their hearing as good, though slightly worse than before (5 or 10 years ago). As far as we could check, the self reports on medical history did not show deviations from the general population.

When categorizing the musicians' pure-tone audiograms in absolute terms, almost half of the tested musicians' ears can be categorized as normal. Among the larger groups (i.e. HS, LS, BW and WW), age seems to be more predictive for audiogram category than the instrument played: the percentage of brass-wind players, who had the lowest average age, was smallest in the sloping-loss category in contrast to the low-string players who had a relatively higher average age and were better represented in this category.

Audiograms corrected for age and gender resulted in better threshold levels for low-string players, as compared to high-string and wood-wind players. This could suggest an effect of exposure as low-string players are usually the least exposed group (Boasson 2002). It was unexpected that the more heavily exposed group (i.e. brass-wind players) did not show a larger increase in the thresholds than the other groups, except for the already mentioned low-string players. All the instrument categories show an evenly profound notch in the hearing-thresholds at $6 \mathrm{kHz}$, a frequency that is known to be very sensitive for noise-induced hearing loss.

When the relative audiometric group results were compared to that of the ISO 7029 (2000) population, musicians showed better hearing thresholds on all tested frequencies, except on $6 \mathrm{kHz}$. This supports the observation that professional musicians perform relatively good on pure-tone audiometry despite intense exposure. It is possible that this effect is able to mask early signs of NIHL and in that case screening techniques other than the pure-tone hearing thresholds could be more adequate for the detection of early stages of NIHL in professional musicians (e.g. Kähäri 2001b). Despite this fact, our material showed that the thresholds at $6 \mathrm{kHz}$ were nevertheless poorer than in the ISO 7029 (2000) population. This finding suggests that at least some hearing loss in musicians can be associated with the duration and intensity of the music that they are exposed to. On the other hand, some studies have come up with the suggestion that deviations at $6 \mathrm{kHz}$, and possibly also at 4 and $8 \mathrm{kHz}$, are caused by shortcomings in the ISO 389 (1991), regarding its representation of hearing threshold levels to be expected in otologically normal adults (see, for example Lutman and Davis 1994). Further research on this matter could lead to different conclusions regarding the $6 \mathrm{kHz}$ notch we found in our musicians' sample.
The second experimental goal was to obtain reliable, objective data on other expressions of noise related hearing problems: hyperacusis, diplacusis, tinnitus, and decreased performance on speech-in-noise tasks. Accordingly, an attempt was made to assess the hearing status of professional musicians more profoundly, not only by specific hearing tests but also by the use of self reports.

Hyperacusis, an increased sensitivity to sound at levels that would normally not be of discomfort to an individual has been associated with exposure to sound and is often reported in people with a known hearing loss (Katzenell and Segal 2001). According to Anari et al. (1999) it occurs in $43 \%$ of musicians. In this study, a large number of musicians indicated to have severe complaints about hyperacusis, but the average UCL values were only slightly lower than that of non-exposed populations. We have to be cautious on this matter as data from other studies are not directly comparable. Keller (2006) found higher average UCL values, but she used different stimuli, and a different procedure to determine these values. Our UCLs were based on noises retrieved from binaural conditions, while Keller used pure tones measured monaurally. Also the UCL was defined in a different way. We found higher UCL-levels at $0.75 \mathrm{kHz}$ NBN than at $3 \mathrm{kHz}$ NBN. This is in disagreement with the results from Keller's study, but corresponds to earlier findings of Morgan et al. (1974). The fact that the dynamic ranges decreased with increasing pure-tone thresholds might indicate some association with NIHL. However the correlation at $6 \mathrm{kHz}$ did not differ from the correlations at other frequencies.

Binaural diplacusis is demonstrated by the fact that two ears of one person each provide a different pitch sensation in response to the same stimulus. In normal hearing ears differences in pitch sensation between 1.6 and $2.3 \%$ with some small variations over time are common (Burns 1982; Brink van den 1982). Only a few very sensitive people experience diplacusis, but also pathological matching of frequency and pitch not experienced by a musician can cause her/him to play out of tune.

A large number of tested musicians had an interaural difference in pitch perception of more than $2 \%$ and the number increased with increasing frequencies. The criterion for the definition of diplacusis used here, an interaural difference of more than $1 \%$, could have been too strict. It is difficult to find evidence on this matter, but in at least one study (Markides 1981) interaural differences of more than $2 \%$ are still considered to be normal. Diplacusis did not seem to cause real problems for musicians, as just a few indicated to struggle with it. On the other hand, musicians with diplacusis had increased average threshold levels while the average age for the groups did not differ, indicating that diplacusis is related to other forms of hearing impairment, possibly NIHL. 
$12 \%$ of men between 65 and 74 of age experience some kind of tinnitus and its prevalence increases with age (Lockwood et al. 2002). In musicians, however, it seems to be far more common. About half of the musicians tested mentioned tinnitus as a complaint. In other studies tinnitus has been reported in 2-20\% (Lockwood et al. 2002; Axelsson et al. 1989; Coles 1984; Skarzyński et al. 2000). The tinnitus reported in this study usually had a temporary character, but some participants reported very loud and continuous tinnitus. In these cases the tinnitus could cause a serious handicap. Tinnitus was more often pitched in the higher frequency area (i.e. higher than $4 \mathrm{kHz}$ ), which strongly suggests that tinnitus is related to intensive exposure to loud sounds. Tinnitus was more often localized utmost left and this could not be related to the instrument type (e.g. in the HS group) or to the position in the orchestra. As with diplacusis, musicians with tinnitus showed increased hearing thresholds, while no difference in age could be found with musicians who did not report tinnitus.

Most musicians scored within normal limits on the speech-in-noise test. The musicians' subjective assessment did not show any severe problems with understanding speech in a noisy environment, or in music.

As the third main theme, we included OAE measurements in order to asses the added value in detection of NIHL and to assess the relations between measurements of hearing acuity (i.e. PTA, OAE) and self-reports on noiseinduced hearing problems.

In both TEOAEs and DPOAEs large inter-individual differences were found. No relation to individual audiometric patterns could be determined. On group level however, we found clear differences between the average OAE responses of different audiometric subgroups: in general, more intense OAEs were found for groups with better average pure-tone thresholds. The OAEs of the normal hearing musicians were clearly distinguishable from the OAEs of the musicians in the other audiometric categories, suggesting a signalling function for early detection of NIHL. A firm statement on this issue can, however, only be made on the basis of a longitudinal study. The dissociation between audiometric thresholds and OAE outcome measures can be a complication in the application of OAEs for screening purposes on an individual level. As long as experimental evidence about the predictive value is not strong enough, the pure-tone audiogram should remain the gold standard for the assessment of NIHL.

Finally, continuing education about the risks of intensive sound exposure to musicians, with the emphasis on the possible development of tinnitus and hyperacusis and the need for good hearing protection (i.e. not only in the form of personal hearing protection such as ear plugs, but also on noise absorbing screens, and the importance of changing position in the orchestra) is warranted.

\section{Conclusions}

In summary, most musicians in this study could be classified as having normal hearing. Relative auditory thresholds were generally better than the normal-hearing reference group of ISO 7029 (2000) standard, except at $6 \mathrm{kHz}$, which clearly suggests an association with NIHL. Tinnitus, diplacusis, and hyperacusis were found more often than could be expected in the general population, based on other studies. Diplacusis does not seem to have much impact on the professional practice of the musicians, but tinnitus and hyperacusis can cause severe problems in professional and private environments. Also the prevalence of tinnitus and diplacusis are suggestive for the involvement of NIHL. Furthermore, to make a statement about the early diagnostic qualities of the otoacoustic emissions towards NIHL, there is a need for more data on the development of otoacoustic emissions over time.

Acknowledgments The authors like to thank Miranda Neerings of the Academic Medical Center Amsterdam for her dedication and accuracy in testing the musicians and prof. J. Festen for giving us the opportunity to use the speech-in-noise-test developed by the VU university medical center. The AMC Medical Ethical Commission approved with this study. This study was supported by the Agency for Dutch Orchestras (Contactorgaan Nederlandse orkesten)

Open Access This article is distributed under the terms of the Creative Commons Attribution Noncommercial License which permits any noncommercial use, distribution, and reproduction in any medium, provided the original author(s) and source are credited.

\section{References}

Anari M, Axelsson A, Eliasson A, Magnusson L (1999) Hypersensitivity to sound: questionnaire data, audiometry and classification. Scand Audiol 28:219-230

Avan P, Bonfils P (1993) Frequency specificity of human distortion product otoacoustic emissions. Audiology 32(1):12-26

Axelsson A, Ringdahl A (1989) Tinnitus: a study of its prevalence and characteristics. Br J Audiol 23(1):53-62

Boasson MW (2002) A one year noise survey during rehearsals and performances in the Netherlands Ballet Orchestra. In: Proceedings of the Institute of Acoustics 24(4):33-34

Brand T, Hohmann V (2002) An adaptive procedure for categorical loudness scaling. J Acoust Soc Am 112(4):1597-1604

Brink van den G (1970) Experiments on bineural diplacusis and tone perception. In: Plomp R, Smoorenburg GF (eds) Frequency analysis and periodicity detection in hearing. Sijthoff, Leiden, pp 362-373

Burns EM (1982) Pure-tone pitch anomalies. I. Pitch-intensity effects and diplacusis in normal ears. J Acoust Soc Am 72(5):1394-1402

Coles RR (1984) Epidemiology of tinnitus: (1) prevalence. J Laryngol Otol Suppl 9:7-15

Coles RR, Lutman ME, Buffin JT (2000) Guidelines on the diagnosis of noise-induced hearing loss for medicolegal purposes. Clin Otolaryngol Allied Sci 25(4):264-273

Dawson-Saunders B, Trapp RG (1994) Basic and clinical biostatistics, 2nd edn. Appleton \& Lange, Connecticut

Dowling wJ, Harwood DL (1986) Music cognition. Academic Press, St Louis 
Eaton S, Gillis H (2002) Review of orchestra musicians hearing loss risks. Can Acoust 30(2):5

Gorga MP, Dierking DM, Johnson TA, Beauchaine KL, Garner CA, Neely ST (2005) A validation and potential clinical application of multivariate analyses of distortion-product otoacoustic emission data. Ear Hear 26:593-607

ISO 389 (1991) Acoustics-standard reference zero for the calibration of pure-tone audiometers, 3rd edn. International organization for standardization, Geneva

ISO 7029 (2000) Acoustics—statistical distribution of hearing thresholds as a function of age, 2nd edn. International organization for standardization, Geneva

Johnson DW, Sherman RE, Aldridge J, Lorraine A (1985) Effects of instrument type and orchestral position on hearing sensitivity for 0.25 to $20 \mathrm{kHz}$ in the orchestral musician. Scand Audiol 14(4):215-221

Kähäri KR, Axelsson A, Hellström PA, Zachau G (2001a) Hearing assessment of classical orchestral musicians. Scand Audiol 30(1):13-23

Kähäri KR, Axelsson A, Hellström PA, Zachau G (2001b) Hearing development in classical orchestral musicians. A follow-up study. Scand Audiol 30(3):141-149

Karlsson K, Lundquist PG, Olaussen T (1983) The hearing of symphony orchestra musicians. Scand Audiol 12(4):257-264

Katzenell U, Segal S (2001) Hyperacusis: review and clinical guidelines. Otol Neurotol 22(3):321-327

Keller JN. (2006) Loudness discomfort levels: a retrospective study comparing data from Pascoe (1988) and Washington University School of Medicine. Washington University School of medicine

Lapsley-Miller JA, Marshall L, Heller LM (2004) A longitudinal study in evoked otoacoustic emissions and pure-tone thresholds as measured in a hearing conservation program. Int $\mathrm{J}$ Audiol 43(6):307-322
Lockwood AH, Salvi RJ, Burkhard RF (2002) Tinnitus. N Engl J Med 347(12):904-910

Lutman ME, Davis AC (1994) The distribution of hearing threshold levels in the general population aged 18-30 years. Audiology 33:327-350

Markides A (1981) Binaural pitch-matching with interrupted tones. $\mathrm{Br}$ J Audiol 15(3):173-180

Martin GK, Ohlms LA, Franklin DJ, Harris FP, Lonsbury-Martin BL (1990) Distortion product emissions in humans. III. Influence of sensorineural hearing loss. Ann Otol Rhinol Laryngol Suppl 147:30-42

Morgan DE, Wilson RH, Dirks DD (1974) Loudness discomfort level: selected methods and stimuli. J Acoust Soc Am 56(2):577-581

Niskar AS, Kieszak SM, Holmes AE, Esteban E, Rubin C, Brody DJ (2001) Estimated prevalence of noise-induced hearing threshold shifts among children 6 to 19 years of age: the Third National Health and Nutrition Examination Survey, 1988-1994, United States. Pediatrics 109(5):987-988

Obeling L, Poulsen T (1999) Hearing ability in Danish symphony orchestra musicians. Noise Health 1(2):43-49

Rabinowitz PM, Galusha D, Slade MD, Dixon-Ernst C, Sircar KD, Dobie RA (2006) Audiogram notches in noise-exposed workers. Ear Hear 27(6):742-750

Seither-Preisler A, Johnson L, Krumbholz K, Nobbe A, Patterson R, Seither S, Lütkenhöner B (2007) Tone sequences with conflicting fundamental pitch and timbre changes are heard differently by musicians and nonmusicians. J Exp Psychol Hum Percept Perform 33(3):743-751

Skarzyński H, Rogowski M, Bartnik G, Fabijańska A (2000) Organization of tinnitus management in Poland. Acta Otolaryngol 12(2):225-226

Smits C, Kapteyn TS, Houtgast T (2004) Development and validation of an automatic speech-in-noise screening test by telephone. Int J Audiol 43(1):15-28 